\title{
Phenotypic Differences between Lactococcus garvieae Isolates Obtained from Rainbow Trout Farms in Turkey
}

\author{
Şükrü Önalan ${ }^{*}$, Muhammed Arabacı ${ }^{1}$ and Haşmet Çă̆ırgan ${ }^{2}$ \\ ${ }^{I}$ Van Yuzuncu Yil University, Fisheries Faculty, Van Yüzüncü Yıl University Turkey \\ ${ }^{2}$ Ege University, Fisheries Faculty, Izmir, Turkey
}

\section{A B S T R A C T}

Lactococcus garvieae is one of the main pathogenic agents in rainbow trout farms in Turkey. Twentytwo $L$. garvieae isolates obtained from different regions in Turkey were evaluated phenotypically in the study. In all isolates, cream colored, bright, round and S-type colonies with smooth margins were seen in TSA medium. They were alive during native examination without movement. It was observed that morphologically all isolates were Gram $(+), \alpha$-hemolytic (BA), oxidase and catalase negative and were reproduced under 21,37 and $45^{\circ} \mathrm{C}$ temperatures with $0-6.5 \% \mathrm{NaCl}$ salinity. As a result of the examination of biochemical properties with API Rapid ID 32 Strep test, it was observed that 2 L. garvieae isolates were different from other isolates in respect of sucrose utilization. 1 and 19 number isolates were negative for sucrose whereas other isolates gave positive results. 1 number isolate was different from other isolates based on maltose profile. While isolate 22 was maltose negative, the other isolates gave positive results. According to phenotypic differences, all isolates used in the study were classified as three different groups.
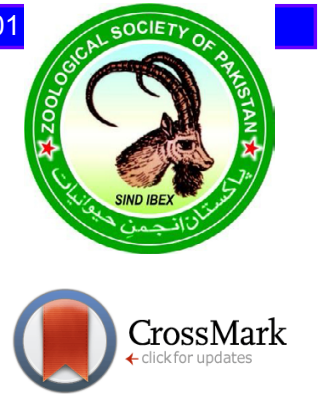

\begin{tabular}{l} 
Article Information \\
\hline Received 18 July 2019 \\
Revised 30 August 2019 \\
Accepted 03 October 2019 \\
Available online 14 May 2020 \\
Authors' Contribution \\
\hline ŞÖ did laboratory and statistical anal- \\
ysis and wrote the manuscript. MA \\
revised the article.HÇ did Lab work \\
and provided isolates. \\
Key words \\
Fish diseases, Lactococcus garvieae, \\
Phenotyping, Rainbow trout, Fish \\
farm
\end{tabular}

\section{INTRODUCTION}

$\mathrm{T}$ he rainbow trout, which is the most cultured fish in the world, is preferred due to the shortness of incubation time, easy adaptation to environmental conditions, high ability to benefit from natural and artificial feed and resistance against diseases compared to other salmonid fishes (Edwards, 1978; Voorhees et al., 2019). In the rainbow trouts of North America, rootstock and business management, certification, hatchery, diseases and marketing are the main problems that challenge the culturing (Arabac1, 2007). Bacterial infections have an important place in fish diseases and Gram-positive cocci have been defined as important fish pathogens in the last decade. Several epidemic and sporadic cases of Grampositive pathogens have been reported in various parts of the world (Arda et al., 2002). As in all the world, one of the main pathogenic factors frequently seen in Turkey in rainbow trout farms is Lactococcus garvieae (Çağırgan, 2009).

L. garvieae, the causative agent of lactococcosis infection, was first isolated in 1960 from yellowtail (Seriola quinqueradiata) fish (Kusuda et al., 1991) and the disease caused by it is called lactococcosis (Austin and Austin, 1999). In Turkey, the disease caused by these

\footnotetext{
* Corresponding author: sukruonalan@yyu.edu.tr 0030-9923/2020/0005-1771 \$9.00/0

Copyright 2020 Zoological Society of Pakistan
}

bacteria was seen for the first time in September 1992 in a small family business in Karacasu, Aydin, the same year five different epizooties were observed in the farm (Çağırgan, 2007). As of 2008, lactococcosis infection has spread to many regions in Turkey (Türe and Altınok, 2012). It is a septicemic disease that causes economic losses in many fish species especially in rainbow trout when water temperature reaches $15^{\circ} \mathrm{C}$ in summer (Diler et al., 2002; Çağırgan, 2004). Sub-classification of the lactococcosisagent $L$. garvieae can be performed separately by phenotypic, serotypic and genotypic techniques, and the differences should be evaluated comparatively to bring together the differences. The lack of information on the different subtypes of the agent reduces the efficacy of vaccines against the disease (Türe and Altınok, 2012). In the present study, the similarities and differences between the biochemical and phenotypic properties were examined in 22 L. garvieae isolates collected from different regions of Turkey at different times.

\section{MATERIALS AND METHODS}

This study was carried out with the permission of the Local Ethics Committee of Animal Experiments of Van Yüzüncü Y1l University on 18.11.2013 and with permission No. 27552122-341. The Study was carried out with a total of 22 isolates including 4 rainbow trouts isolated from farms located in Van, Bitlis, Muş and Hakkari and $18 \mathrm{~L}$. garvieae isolates collected from different regions in Turkey. 
The rainbow trout samples were brought to the laboratory at $4{ }^{\circ} \mathrm{C}$ on the same day and necropsy was performed under aseptic conditions. Inoculations made from kidney tissues of fish samples were incubated in TSA medium under aerobic conditions at $21^{\circ} \mathrm{C}$ for $24 \mathrm{~h}$. The study was carried out with the bacteria that match the colony morphology of L. garvieae following the incubation period.

Gram staining of the isolates, a drop of $0.6 \%$ FTS was added on the slides from pure bacteria which were produced as a result of the $24-48$-h incubation at $21^{\circ} \mathrm{C}$ in TSA medium. Subsequently, the procedure was completed by treatment with crystal violet for $1 \mathrm{~min}$, glycol for $1 \mathrm{~min}$, alcohol for $15 \mathrm{sec}$ and safranin for $1 \mathrm{~min}$. Preparations were left to dry and then examined with binocular microscope (Leica ICC50 HD) with 4X, 10X, 40X and 100X magnifications, respectively. Following Gram staining, the isolates that were blue-violet color were evaluated as $\mathrm{Gr}(+)$ while red-pink color isolates were evaluated as $\mathrm{Gr}$ (-) (Beşe, 1974).

Bactident Oxidase commercial kit was used for the oxidase test. After $24-48 \mathrm{~h}$-incubation period at $21^{\circ} \mathrm{C}$ in TSA medium, samples from the formed colonies was taken using a sterile loop and spread on sterile filter paper was wetted with oxidase kit. The color changes on the paper surface within $30 \mathrm{sec}$ were examined. Blue color formation was evaluated as positive, and no color formation was considered as oxidase negative (Çă̆ırgan, 2007).

For the catalase test, 1-2 drops of 3\% hydrogen peroxide was added to the slides and mixed with the sample taken using a sterile loop from the colonies grown on TSA medium at $21^{\circ} \mathrm{C}$ for $24-48 \mathrm{~h}$ after incubation. The gas-forming isolates were determined as catalase-positive and the isolates that did not show any gas formation were evaluated as catalase negative (Austin and Austin, 1999).

As a result of the incubation of isolates at $21{ }^{\circ} \mathrm{C}$ for 24-48 $\mathrm{h}$, the pure bacterial isolates were inoculated in sheep blood agar for hemolysis test. After the incubation period, bacteria were examined for their hemolytic properties. Hemolysis types were determined according to the green zone areas formed around the bacteria growing in the medium (Türe, 2011).

Tolerance to different salinity ratios were tested to determine the physiological characteristics of the isolates. Accordingly, sterile TSA media ( $\mathrm{pH}:$ 7.4) containing 4\% and $6.5 \% \mathrm{NaCl}$ were prepared and incubated at $21{ }^{\circ} \mathrm{C}$ for 24-48 h (Beşe, 1974).

The bacteria that were inoculated in Petri dishes containing TSA medium prepared under sterile conditions were enumerated to determine their reproductive ability as a result of incubation at $21{ }^{\circ} \mathrm{C}, 37^{\circ} \mathrm{C}$ and $45^{\circ} \mathrm{C}$ for $24 \mathrm{~h}$ for temperature tolerance test (Kurtoğlu and Korun, 2018).
To determine the biochemical properties of isolates, API Rapid ID 32 Strep (Biomerieux, France) test was adopted. Pure L. garvieae isolates obtained in TSA medium were incubated in BA medium at $24{ }^{\circ} \mathrm{C}$ for $24 \mathrm{~h}$ and the colonies taken by the swap method and bacteria samples were added into tubes containing $2 \mathrm{~mL}$ sterile water until McFarland 4 turbidity was obtained. The suspension solution was prepared for immediate use in the ID 32 strep assay. Bacteria suspension was incubated by adding 55 ul to each well. The results were evaluated in accordance with the manufacturer's specifications.

The positive and negative data of all reagents in the API kit, in which the biochemical differences were determined, were evaluated using the SPSS 20 (IBM, Inc) package program as binomial data.

\section{RESULTS AND DISCUSSION}

It was seen that 24 of 54 rainbow trout farms that were visited for sampling were closed for various reasons in the summer of 2014 (June-July-August-September). The production capacity of the 30 rainbow trout farms was between 1 and 500 tons. During the summer months, the temperature during the sample collection procedure was between $19^{\circ} \mathrm{C}$ and $38^{\circ} \mathrm{C}$, while the water temperature in rainbow trout farms where the samplings were carried out was between $13{ }^{\circ} \mathrm{C}$ and $24^{\circ} \mathrm{C}$. In the sampled fish, ascites, darkening of skin and exophthalmos were observed in fish. Four L. garvieae isolates from these fish farms were obtained. Also, 18 L. garvieae isolates were included in study from Turkey's various regions. This study was carried out with 22 isolates. Gram staining revealed that all isolates were coccus-shaped Gram positive. As a result of salinity tolerance tests, 22 isolates were able to grow in $4 \%$ and $6.5 \%$ salinity ratios in TSA medium.

To determine the phenotypic differences of isolates, API Rapid ID 32 Strep rapid diagnostic test was evaluated according to the evaluation table of the manufacturer (Biomerioux). As a result of API Rapid ID 32 Strep evaluation table, isolates 1 and 19 gave negative results in contrast to other isolates in terms of sucrose (red color; negative), other phenotypic features showed similar profiles with all isolates. The isolate 22 was separated from the other isolates (positive) by giving the maltose profile red-orange color (negative). Differences were observed in the phenotypic profiles of L. garvieae isolates obtained as a result of the API test. A total of three different phenotypic profiles were formed including API profiles "30321111020" for isolate 1 and isolate 19, API profile "30321110120" for Isolate 20, and API profiles for other isolates “30321111120” (Table I). 
Table I. Biochemical properties and differences of L.garvieae isolates with API rapid ID 32 strep.

\begin{tabular}{|c|c|c|c|c|c|c|c|c|c|c|c|c|c|c|c|c|c|c|c|c|c|c|c|}
\hline \multirow{3}{*}{$\begin{array}{l}\text { Testin } \\
\text { Kodu }\end{array}$} & \multirow{3}{*}{$\begin{array}{l}\text { İzolat Adı } \\
\text { Testin Adı } \\
\end{array}$} & \multirow{3}{*}{$\begin{array}{l}\text { Fenotip I } \\
1\end{array}$} & \multicolumn{17}{|c|}{ Fenotip III } & \multirow[t]{3}{*}{ Fenotip I } & \multicolumn{2}{|c|}{$\begin{array}{c}\text { Fenotip } \\
\text { III }\end{array}$} & \multirow{3}{*}{$\begin{array}{c}\begin{array}{c}\text { Fenotip } \\
\text { II }\end{array} \\
22\end{array}$} \\
\hline & & & \multirow[t]{2}{*}{2} & \multirow[t]{2}{*}{3} & \multirow[t]{2}{*}{34} & \multirow[t]{2}{*}{5} & \multirow{2}{*}{\multicolumn{2}{|c|}{6}} & \multirow[t]{2}{*}{8} & \multirow[t]{2}{*}{9} & \multirow[t]{2}{*}{1} & \multirow[t]{2}{*}{011} & 12 & 13 & 14 & 15 & 16 & 17 & 18 & & 20 & 21 & \\
\hline & & & & & & & & 7 & & & & & & & & & & & & & & & \\
\hline 1.0 & $\mathrm{ADH}$ & + & + & + & + & + & + & + & + & + & + & + & + & + & + & + & + & + & + & + & + & + & + \\
\hline 1.1 & BGLU & + & + & + & + & + & + & + & + & + & + & + & + & + & + & + & + & + & + & + & + & + & + \\
\hline 1.2 & BGAR & - & - & - & - & - & - & - & - & - & - & - & - & - & - & - & - & - & - & - & - & - & - \\
\hline 1.3 & BGUR & - & - & - & - & - & - & - & - & - & - & - & - & - & - & - & - & - & - & - & - & - & - \\
\hline 1.4 & aGAL & - & - & - & - & - & - & - & - & - & - & - & - & - & - & - & - & - & - & - & - & - & - \\
\hline 1.5 & PAL & - & - & - & - & - & - & - & - & - & - & - & - & - & - & - & - & - & - & - & - & - & - \\
\hline 1.6 & RIB & + & + & + & + & + & + & + & + & + & + & + & + & + & + & + & + & + & + & + & + & + & + \\
\hline 1.7 & MAN & + & + & + & + & + & + & + & + & + & + & + & + & + & + & + & + & + & + & + & + & + & + \\
\hline 1.8 & SOR & - & - & - & - & - & - & - & - & - & - & - & - & - & - & - & - & - & - & - & - & - & - \\
\hline 1.9 & LAC & - & - & - & - & - & - & - & - & - & - & - & - & - & - & - & - & - & - & - & - & - & - \\
\hline 1.A & TRE & + & + & + & + & + & + & + & + & + & + & + & + & + & + & + & + & + & + & + & + & + & + \\
\hline 1.B & RAF & - & - & - & - & - & - & - & - & - & - & - & - & - & - & - & - & - & - & - & - & - & - \\
\hline 1.C & SAC & - & + & + & + & + & + & + & + & + & + & + & + & + & + & + & + & + & + & - & + & + & + \\
\hline & & $\bullet$ & & & & & & & & & & & 0 & & & & & & & 0 & & & \\
\hline 1.D & LARA & - & - & - & - & - & - & - & - & - & - & - & - & - & - & - & - & - & - & - & - & - & - \\
\hline 1.E & DARL & - & - & - & - & - & - & - & - & - & - & - & - & - & - & - & - & - & - & - & - & - & - \\
\hline 1.F & CDEX & - & - & - & - & - & - & - & - & - & - & - & - & - & - & - & - & - & - & - & - & - & - \\
\hline 0.0 & VP & + & + & + & + & + & + & + & + & + & + & + & + & + & + & + & + & + & + & + & + & + & + \\
\hline 0.1 & APPA & - & - & - & - & - & - & - & - & - & - & - & - & - & - & - & - & - & - & - & - & - & - \\
\hline 0.2 & bGAL & - & - & - & - & - & - & - & - & - & - & - & - & - & - & - & - & - & - & - & - & - & - \\
\hline 0.3 & PyrA & + & + & + & + & + & + & + & + & + & + & + & + & + & + & + & + & + & + & + & + & + & + \\
\hline 0.4 & bNAG & - & - & - & - & - & - & - & - & - & - & - & - & - & - & - & - & - & - & - & - & - & - \\
\hline 0.5 & GTA & - & - & - & - & - & - & - & - & - & - & - & - & - & - & - & - & - & - & - & - & - & - \\
\hline 0.6 & HIP & + & + & + & + & + & + & + & + & + & + & + & + & + & + & + & + & + & + & + & + & + & + \\
\hline 0.7 & GLYG & - & - & - & - & - & - & - & - & - & - & - & - & - & - & - & - & - & - & - & - & - & - \\
\hline 0.8 & PUL & - & - & - & - & - & - & - & - & - & - & - & - & - & - & - & - & - & - & - & - & - & - \\
\hline 0.9 & MAL & + & + & + & + & + & + & + & + & + & + & + & + & + & + & + & + & + & + & + & + & + & - \\
\hline & & 0 & & & & & & & & & & & & & & & & & & & & & $\bullet$ \\
\hline $0 . \mathrm{A}$ & MEL & - & - & - & - & - & - & - & - & - & - & - & - & - & - & - & - & - & - & - & - & - & - \\
\hline $0 . \mathrm{B}$ & MLZ & - & - & - & - & - & - & - & - & - & - & - & - & - & - & - & - & - & - & - & - & - & - \\
\hline $0 . \mathrm{C}$ & M8DG & - & - & - & - & - & - & - & - & - & - & - & - & - & - & - & - & - & - & - & - & - & - \\
\hline 0.D & TAG & + & + & + & + & + & + & + & + & + & + & + & + & + & + & + & + & + & + & + & + & + & + \\
\hline 0.E & bMAN & - & - & - & - & - & - & - & - & - & - & - & - & - & - & - & - & - & - & - & - & - & - \\
\hline $0 . \mathrm{F}$ & URE & - & - & - & - & - & - & - & - & - & - & - & - & - & - & - & - & - & - & - & - & - & - \\
\hline
\end{tabular}

*Red, negative results; Yellow, positive results; these results are differences between different $L$. garvieae isolates.

In the present study, to isolate L. garvieae strains, inoculations were carried out from kidney tissues. Previous studies have also reported that they used TSA for isolation from kidney tissue samples in their studies on lactococcosis (Holt et al., 1994; Toranzo et al., 1994; Eldar et al., 1996, 1999; Buller, 2004; Cagirgan, 2004; 
Vendrell et al., 2006). Some researchers have reported that they used BHIA, biliary agar (BA) and biliary eculin agar (BEA) media together with TSA for the isolation of L. garvieae (Türe, 2011; Ürkü, 2011). However, Ürkü (2011), in her study, examined the histopathological findings of spleen and liver tissues as well as kidney tissue and reported the same results on BHIA and TSA media. All of the L. garvieae strains were found to have a cream-colored, round shaped and S-type colony structure in terms of colony morphologies. It was determined that the results had characteristics of L. garvieae in terms of morphological properties. In parallel with the results of the present study, the previous researchers have reported that L. garvieae strains obtained from rainbow trout have cream-colored, round and S-type colony structure in TSA (Buller, 2004; Cagirgan, 2004; Ürkü, 2011; Vendrell et al., 2006; Kurtoğlu and Korun, 2018). All L. garvieae strains were found to be $\alpha$-hemolytic in $5 \%$ sheep blood agar. In line with the results of the present study, previous studies have reported that L. garvieae strains obtained from rainbow trout had $\alpha$-hemolytic structure (Buller, 2004; Cagirgan, 2004; Vendrell et al., 2006; Tanrıkul and Gültepe, 2011). Çağırgan (2007) have reported that some of the L. garvieae strains have $\alpha$-hemolitik structure while some have non-hemolytic structure. The $\alpha$-hemolytic structure of $L$. garvieae strains used in this study suggests that these strains may be more pathogenic. Some researchers described $L$. garvieae strains as $\beta$-hemolytic (Teixeira et al., 1996). Teixera et al. (1996) have reported different hemolytic properties in L. garvieae strains. This can be associated with different isolation sources (buffalo) of L. garvieae isolates. However, the differences between the methods used in the hemolysis test of streptococci are important. All of the L. garvieae strains used in the study showed salinity tolerance test in the salinity ratios of $4 \%$ and $6.5 \% \mathrm{NaCl}$ in TSA.

Previous researchers have also reported that $L$. garvieae strains obtained from rainbow trout showed growth in media containing $4 \%$ or $6.5 \% \mathrm{NaCl}$ (Eldar et al., 1999; Vela et al., 2000; Buller, 2004; Kawanishi et al., 2005; Vendrell et al., 2006; Kurtoğlu and Korun, 2018). As a result of the study, the salinity tolerance rates of $L$. garvieae strains were found to be similar with those previously reported. All of the L. garvieae strains obtained in the study were observed could grow in TSA at temperatures 21,37 and $45^{\circ} \mathrm{C}$. The strains exhibited a lesser growth at $37^{\circ} \mathrm{C}$ and $45^{\circ} \mathrm{C}$ in TSA compared to that at $21^{\circ} \mathrm{C}$. The optimum temperature for the API (Biomerioux) test is reported in the package insert at $37^{\circ} \mathrm{C}$. Previous researchers have shown that $L$. garvieae strains show growth at $21{ }^{\circ} \mathrm{C}, 37{ }^{\circ} \mathrm{C}$ and $45{ }^{\circ} \mathrm{C}$, in parallel with the results of the present study (Kusuda et al., 1991; Prieta et al., 1993; Eldar et al., 1996; Fortina et al., 2007) whereas some researchers have reported that L. garvieae strains showed poor growth at $45{ }^{\circ} \mathrm{C}$ (Teixeira et al., 1996). To determine the phenotypic properties of $L$. garvieae strains, API Rapid ID 32 Strep test was used by many researchers (Eldar et al., 1999; Vela et al., 2000; Baeck et al., 2006; Altun et al., 2007; Türe and Altınok, 2012; Altun et al., 2013). Following the API Rapid ID 32 Strep test to determine biochemical properties and differences between isolates, different phenotypic profiles were formed due to the reaction of sucrose (SAC) and maltose (MAL) reagents. Considering these differences, L. garvieae strains were found to form three different phenotypic groups. These groups were evaluated as those using sucrose $(+)$ and not using maltose (-) phenotype-1, non-sucrose (-) and maltose $(+)$ phenotype- 2 and phenotype- 3 using both reagents. In parallel with the results of the present study, Eldar et al. (1999) reported that L. garvieae strains obtained from rainbow trout showed differences in terms of tagatose (TAG) and sucrose (SAC) tests as a result of API Rapid ID 32 Strep test. Eldar et al. (1999) have grouped the strains obtained from rainbow trout farms in Italy and ATCC reference strains as phenotype-1 (TAG: -, SAC: -), the only strain from the rainbow trout farm in Australia as phenotype-2 (TAG: +, SAC: -), and strains obtained from rainbow trout farms in Spain (TAG: +, SAC: + ) as phenotype- 3 . The phenotype-3 group obtained in this study was similar to phenotype- 3 group reported by Eldar et al. (1999), while phenotype-2 group was similar to phenotype-2 group. Eldar et al. (1999), similar to the results obtained in the present study, have reported that they obtained three phenotypic groups, however only two groups (phenotype-2 and phenotype-3) were found to have phenotypically common properties between the two studies. This difference can be associated with collecting strains from different locations and the difference between the number of strains studied. Çağırgan (2004) performed API CH 50 test at $24^{\circ} \mathrm{C}$ to determine phenotypic differences between 20 isolated L. garvieae strains from rainbow trout farms located in various regions of in Turkey. Çağırgan (2004) have reported that phenotypic differences in $L$. garvieae strains in terms of acid formation from lactose and, as a result of these differences, L. garvieae strains produced 2 different phenotypic profiles. Çağırgan (2004) compared the results they obtained to those by Eldar et al. (1999) and reported that phenotype-2 group was phenotypically similar to Spanish strains. The phenotype-2 obtained by Çağırgan (2004) and the phenotype-3 group obtained from the present study were similar, therefore the phenotype-3 group obtained in the present study was similar to the Spanish strains. Altun et al. (2004) collected 4 L. garvieae strains from rainbow trout farms in Turkey, 
2 from rainbow trout farms in Spain, 1 from rainbow trout farms in England and 1 reference strain (ATCC 43921), a total of 8 strains, and determined their phenotypic differences with API Rapid ID 32 Strep test at $37^{\circ} \mathrm{C}$. Altun et al. (2004) reported that mannitol (MAN), tagatose (TAG) and sucrose (SAC) results were similar and positive in all strains after phenotypic examinations. Evaluating the phenotypic results, it was seen that phenotypic properties of strains obtained from rainbow trout by Altun et al. (2004) were similar to the phenotype-3 group obtained in the present study. Türe (2011) phenotypically examined a total of 41 L. garvieae strains, 3 obtained from rainbow trout, Black Sea trout and sea bass samples from Turkey, and 38 obtained by different researchers Italy, France and Japan. The phenotypic differences between the L. garvieae strains were investigated at $30^{\circ} \mathrm{C}$ using API 20 Strep kit. As a result of the API 20 Strep test, differences were reported in terms of Voges Proskauer (VP), hippurate (HIP), pyroglutamic acid arylamidase (PyrA), $\alpha$-galactosidase $(\alpha-\mathrm{GAL}), \beta$-galactosidase (b-GAL), arginine dhidrolase $(\mathrm{ADH})$, ribose (RIB), mannitol (MAN), lactose (LAC) and trehalose (TRE) reagents. Türe (2011) have reported that L. garvieae strains formed 8 different phenotypic groups. The phenotype- 2 obtained by Türe (2011) and the phenotype- 3 group obtained in the present study had similar properties. Differences in the phenotypic groups between those reported by Türe (2011) and the present study was associated with the different types of API kits used and L. garvieae strains isolated from different sources (rainbow trout, sea bass and Black sea trout). Türe (2011) used API 20 Strep tests at $30{ }^{\circ} \mathrm{C}$ incubation temperature whereas, API Rapid ID 32 Strep tests was used at $37{ }^{\circ} \mathrm{C}$ incubation temperature in the present study. Vela et al. (2000) phenotypically examined $L$. garvieae strains they obtained from rainbow trout in Portugal, France and Italy, from buffalo and humans in Brazil, from humans in the USA, from yellowtail fish in Japan, and from cattle and rainbow trout in Spain. Unlike the present study, Vela et al. (2000) used API Rapid ID 32 Strep at $30^{\circ} \mathrm{C}$, and reported that there were differences between the L. garvieae strains in terms of sucrose (SAC), tagatose (TAG), mannitol (MAN) use and cyclodextrin (CDEX), pyroglutamic acid arylamidase (PyrA) and $\mathrm{N}$-acetyl- $\beta$-glucosaminidase $(\beta \mathrm{NAG})$ reagents. As a result of these differences, it was reported that L. garvieae strains formed 13 phenotypic groups. The phenotype-I group obtained from rainbow trout by Vela et al. (2000) were similar to phenotype-1 and phenotype-3 groups obtained in the present, furthermore phenotype-3 group obtained by the researchers were similar to phenotype-2 group obtained in the present study. The other 11 phenotypic groups obtained by Vela et al. (2000) were not similar to those obtained in the present study. This difference was associated with the fact that Vela et al. (2000) isolated L. garvieae strains that form 13 different phenotypes from different sources (water, human, cattle, fish and buffalo) and used API Rapid ID 32 Strep tests at $30^{\circ} \mathrm{C}$. In the present study, L. garvieae strains were isolated only from rainbow trout and incubated in API Rapid ID 32 Strep test at $37^{\circ} \mathrm{C}$.

Altun et al. (2013) identified the biochemical properties of L. garvieae strains with API Rapid ID 32 Strep, similar to the method used in the present study. In terms of phenotypic features the researchers have reported that they obtained positive results from $\beta$-glucosidase (b-GUL), ribose (RIB), sorbitol (SOR), lactose (LAC), raffinose (RAF), Voges Proskauer (VP), alanyl-phenylalanyl-proline arylamidase (APPA), pyroglutamic acid arylamidase (PyrA), hippurate (HIP) and urease (URE) reagents. Although the sucrose (SAC) and mannitol (MAN) reagent results obtained by Altun et al. (2013) as a result of API Rapid ID 32 Strep test was similar to the results of the study, no similar groups were determined in terms of phenotypic groups. API kits used in the present study and by Altun et al. (2013) and were the same, however different incubation temperatures may be the reason why different phenotypic groups were obtained. Altun et al. (2007) incubated API Rapid ID 32 strep test at $37{ }^{\circ} \mathrm{C}$ and reported similar groups with phenotypic groups obtained in the present study. However, the groups obtained by Altun et al. (2013) by using the API Rapid ID 32 strep test at $35{ }^{\circ} \mathrm{C}$ were not similar to those obtained in the present study. Detailed research is needed to clarify the reasons for these differences. In this study, similar groups were observed between phenotypic groups formed by L. garvieae strains in the present study and the previous studies. It was found that phenotype-2 group obtained by Eldar et al. (1999) from rainbow trout was similar to phenotype-2 obtained in the present study while phenotype-3 group obtained by Eldar et al. (1999) also from rainbow trout was similar to phenotype-3 group obtained in the present study. The phenotype-I group obtained from rainbow trout by Vela et al. (2000) were similar to phenotype- 1 and phenotype- 3 groups obtained in the present study. Furthermore, phenotype-3 group obtained by the researchers were similar to phenotype- 2 group obtained in the present study. It was seen that phenotype-1 group of strains obtained from rainbow trouts by Altun et al. (2007) were similar to the phenotype-3 group obtained in the present study. phenotype- 2 group obtained from rainbow trout by Çağırgan (2004) was similar to the phenotype- 3 group obtained in the present study. The phenotype-2 group obtained from $L$. garvieae strains by Türe (2011) was similar to the phenotype-3 group obtained in the present study. Comparing the phenotypic test results 
obtained in the present study with those obtained in the previous studies, the differences can be associated with different sources (human, bovine, buffalo, cat, chicken, water, Black sea trout and sea bass) and the application of tests used to characterize the biochemical characteristics of strains under different environmental conditions (incubation temperature). Studies on the effects of different environmental conditions on results are needed. It is thought that evaluating all of the L. garvieae strains obtained by the previous studies together and under same conditions will yield better results in the determination of the phenotypic differences in L. garvieae strains.

The results of the present study showed that L. garvieae is one of the main pathogenic agents in rainbow trout farms in Turkey. Twenty two L. garvieae isolates obtained from different regions in Turkey were evaluated phenotypically in the study. In all isolates, cream colored, bright, round and S-type colonies with smooth margins were seen in TSA medium. They were alive during native examination without movement. It was observed that morphologically all isolates were Gram (+), $\alpha$-hemolytic (BA), oxidase and catalase negative and were reproduced under 21 , 37 and $45{ }^{\circ} \mathrm{C}$ temperatures with $0-6.5 \% \mathrm{NaCl}$ salinity. As a result of the examination of biochemical properties with API Rapid ID 32 Strep test, it was observed that 2 L. garvieae isolates were different from other isolates in respect of sucrose utilization. One and 19 number isolates were negative for sucrose whereas other isolates gave positive results. $1 \mathrm{~L}$. garvieae isolate was different from other isolates based on maltose profile. While isolate 22 was maltose negative, the other isolates gave positive results. According to phenotypic differences, all isolates used in the study were classified as 3 different groups (Table II).

Table II. Phenotypic groups of $L$. garvieae isolates and their properties.

\begin{tabular}{llll}
\hline & SAC & MAL & Isolate number \\
\hline Phenotype-1 & - & + & 1,19 \\
Phenotype -2 & + & - & 22 \\
Phenotype -3 & + & + & Other 19 isolates \\
\hline
\end{tabular}

\section{ACKNOWLEDGEMENTS}

This research was supported by Research Fund of the Van Yüzüncü Yil University. Project Number: 2015-VFB179.

\section{Statement of conflict of interest}

The authors declare there is no conflict of interest.

\section{REFERENCES}

Altun, S., Adiloğlu, A.K., Kubilay, A., Diler, O., Delibaş, N. and Sutcu, R., 2007. Immunogenic and antigenic profiles of nine $L$. garvieae strains from different rainbow trout farms. Israeli J. Aquacult.Bamidgeh, 59: 111-116.

Altun, S., Diler, Ö. and Adiloğlu, K., 2004. Genotyping of L. garvieae strains from rainbow trout (Oncorhynchus mykiss) by 16s rDNA sequencing. Bull. Eur. Assoc. Fish Pathol., 24: 119.

Altun, S., Onuk, E.E., Çiftçi, A., Büyükekiz, A.G. and Duman, M., 2013. Phenotypic, genotypic characterisation and antimicrobial susceptibility determination of L. garvieae strains. Kafkas Univ. Vet. Fak. Derg., 19: 375-381. https://doi. org $/ 10.9775 / \mathrm{kvfd} .2012 .7754$

Arabac1, M., 2007. Rainbow trout farming. Eastern Anatolia Development Program, Agriculture and Rural Development Component. pp. 6.

Arda, M., Seçer, S. and Sarıeyyüpoglu, M., 2002. Fish diseases. Medisan Publishing Series, 1st Edition, Ankara.

Austin, B. and Austin, D.A., 1999. Bacterial fish pathogens: Disease in farmed and wild fish. $3^{\text {rd }}$ (Revised) Edition. Praxis Publishing, Chichester, UK.

Baeck, G.W., Kim, J.H., Gomez, D.K. and ve Park, S.C., 2006. Isolation and Characterization of Streptococcus sp. from diseased flounder (Paralichthys olivaceous) in Jeju Island. J. Vet. Sci., 7: 53-58. https://doi.org/10.4142/jvs.2006.7.1.53

Beşe, M., 1974. Used in microbiology biochemical tests and media. Ankara Univ. Vet. Faculae. Publications, pp. 298.

Buller, N.B., 2004. Bacteria from fish and other aquatic animals: A practical identification manual. CABI Publishing, UK., 99-129. https://doi. org/10.1079/9780851997384.0000

Çă̆ırgan, H., 2004. Biotyping of Lactococcus garvieae isolated from Turkey. Ege Üniversitesi Su Ürünleri Dergisi. J. Fish. Aquat. Sci., 21: 3-4, 267-269.

Çağırgan, H., 2007. Rainbow trout diseases. Eastern Anatolia Development Program, Agriculture and Rural Development Component.

Çağırgan, H., 2009. The use of veterinary drugs and vaccines in Turkey. In: Options Méditerranéennes (eds. C. Rogers C. and B. Basurco). Série A, Séminaires Méditerranéens. pp. 29-34.

Diler, O., Altun, S., Adiloglu, A., Kubilay, A. and Işıklı, B., 2002. First occurance of streptococcosis affecting farmed rainbow trout in Turkey. Bull. Eur. 
Assoc. Fish Pathol., 22: 21-26.

Edwards, D.J., 1978. Salmon and trout farming in Norway, Farnham and England. Fishing News Books Ltd.

Eldar, A., Ghittino, C., Asanta, L., Bozetta, E., Goria, M., Prearo, M. and Bercovier, H., 1996. Enterococcus seriolicida is a Junior Synonym of L. garvieae, a causative agent of septicemia and meningoencephalitis in fish. Curr. Microbiol., 32: 85-88. https://doi.org/10.1007/s002849900015

Eldar, A., Goria, M., Ghittino, C., Zlotkin, A. and ve Bercovier, H., 1999. Biodiversity of L. garvieae strains isolated from fish in Europe, Asia, and Australia. Appl. environ. Microbiol., 65: 10051008.

Fortina, M.G., Ricci, G., Foschino, R., Picozzi, C., Dolci, P., Zeppa, G., Cocolin, L. and Manachini, P.L., 2007. Phenotypic typing, technological properties and safety aspects of $L$. garvieae strains from dairy environments. J. appl. Microbiol., 103: 445-453. https://doi.org/10.1111/j.1365-2672.2006.03265.x

Holt, J.G., Krieg, N.R., Sneath, P.H.A. and Williams, S.T., 1994. Bergey's manual of determinative bacteriology. Immunol. Microbiol. Infect. Dis., 29: 177-198.

Kawanishi, A., Kojima, A., Ishihara, K., Esaki, H., Kijima, M. and ve Takahashi, T., 2005. Drug resistance and pulsed-field gel electrophoresis patterns of L. garvieae isolates from cultured Seriola (Yellowtail, Amberjack and Kingfish) in Japan. Lett. appl. Microbiol., 40: 322-328. https:// doi.org/10.1111/j.1472-765X.2005.01690.x

Kurtoğlu, M. and Korun, J., 2018. Yavru Gökkuşağ 1 alabalıkları (Oncorhynchus mykiss, W.)'ndan Lactococcus garvieae'nin İzolasyonu ve Plazmit Profilleri Üzerine Bir Çalışma. J. Adv. Vet. Biol. Sci. Technol., 3: 11-22. https://doi.org/10.31797/ vetbio. 401284

Kusuda, K., Kawai, K., Salati, F., Banner, C.R. and Freyer, J.L., 1991. Enterococcus serolocida sp. nov, a fish pathogen. Int. J. System. Bact., 41: 406409. https://doi.org/10.1099/00207713-41-3-406

Prieta, J., Domenech, A.M., Fernandez-Garaizabal, J.F., Collins, M.D., Rodrigues, U.M. and Jones, D., 1993. Laktokokkozis in rainbow trout (Oncorhynchus mykiss). Med. Vet., 10: 367-373.

Tanrıkul, T.T. and Gültepe, N., 2011. Mix infection in rainbow trout (Oncorhynchus mykiss, Walbaum) $L$. garvieae and Vibrio anguillarum O1. J. Anim. Vet. $A d v, 10$ (8):1019-1023. https://doi.org/10.3923/ javaa.2011.1019.1023

Teixeira, L.M., Merquior, V.L., Vianni, M.C., Carvalho, M.G., Fracalanzza, S.E. and Steigerwaltz, A.G., 1996. Phenotypic and genotypic characterization of atypical L. garvieae strains isolated from water buffalos with subclinical mastitis and confirmation of L. garvieae as a senior subjective synonym of Enterococcus seriolicida. Int. J. Syst. Bact., 46: 664-668. https://doi.org/10.1099/00207713-46-3664

Toranzo, A.E., Devesa, S., Heinen, P., Riaza, A., Nunez, S. and Barja, J.L., 1994. Streptococcosis in cultured Turbot caused by an enterococcus-like Bacterium. Bull. Eur. Ass. Fish Pathol., 14: 19-23.

Türe, 2011. Phenotypic and genetics of L. garvieae determination of diversity. MSc thesis, Fisheries Technology Engineering Department, Karadeniz Technical University.

Türe, M., ve Altınok, İ., 2012. PFGE method genetic diversity of $\mathrm{L}$. garvieae using and determination of propagation. Seafood Central Research InstituteTrabzon. TAGEM/HS/10/09/02) 179.

Ürkü, Ç., 2011. In rainbow trout (Oncorhynchus mykiss, W.) experimentally bacteriological and lactococcosis created diagnosis with serological methods. Master thesis, Istanbul University, Institute of Science.

Vela, A.I., Vazquez, J., Gibello, A., Blanco, M.M., Moreno, M.A. and ve Liebana, P., 2000. Phenotypic and genetic characterization of $L$. garvieae isolated in Spain from laktokokkozis outbreaks in comparison with isolates of other countries and sources. J. clin. Microbiol., 38: 3791-3795.

Vendrell, D., Balcazar, J.L., Ruiz Zarzuela, I., Blas, I., Girones, O. and Muzquiz, J.L., 2006. L. garvieae in fish: A review. Comp. Immunol. Microbiol. Infect. Dis., 29: 177-98. https://doi.org/10.1016/j. cimid.2006.06.003

Voorhees, J.M., Barnes, M.E., Chipps, S.R. and Brown, M.L., 2019. Bioprocessed soybean meal replacement of fish meal in rainbow trout (Oncorhynchus mykiss) diets. Cogent Fd. Agric., Article number: 1579482. https://doi.org/10.1080/ 23311932.2019.1579482 